\title{
Polarimetric and Bragg Optical Fiber Sensors for Stress Distribution and Temperature Measurements in Composite Materials
}

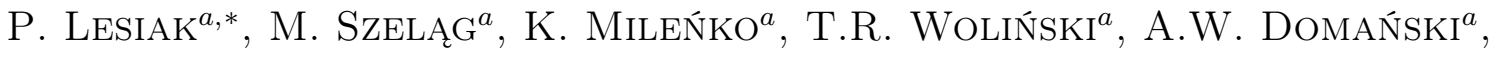 \\ K. JĘDRZEJEWSKI ${ }^{b}$ AND A. BOCZKOWSKA ${ }^{c}$ \\ ${ }^{a}$ Faculty of Physics, Warsaw University of Technology, Koszykowa 75, 00-662 Warsaw, Poland \\ ${ }^{b}$ Faculty of Electronics and Information Technology, Warsaw University of Technology \\ Nowowiejska 15/19, 00-665 Warsaw, Poland \\ ${ }^{c}$ Faculty of Materials Science and Engineering, Warsaw University of Technology \\ Wołoska 141, 02-507 Warsaw, Poland
}

\begin{abstract}
Composite structures are made from two or more constituent materials with significantly different physical or chemical properties and they remain separate and distinct in a macroscopic level within the finished structure. This feature allows us for introducing an optical fiber sensors matrix into the composite material. These sensors can demonstrate stress distribution inside a tested material influenced by external tensions. Two types of the optical fiber sensors, placed into one fiber simultaneously, are used as $s$ matrix structure. One of them is based on application of the Bragg grating structure written inside the core of the fiber. Longitudinal stress modifies changes parameters of the Bragg grating and in the same, spectral characteristics of the light transmitted through the fiber. The second one is based on application of highly birefringent fibers which under external stress introduce polarization changes in the output light. These sensors placed into one fiber give a possibility to the measure two external tensions separately.
\end{abstract}

PACS: 42.81.Gs, 42.81.Pa, 62.23.Pq

\section{Introduction}

Polarimetric optical fiber sensors based on highly birefringent $(\mathrm{HB})$ polarization-maintaining fibers have focused great interest for last decades $[1,2]$. In HB fibers, the difference between the phase velocities for the two orthogonally polarized modes is high enough to avoid coupling between these two modes. Fibers of this class have a built-in, well-defined, high internal birefringence obtained by designing a core and/or cladding with noncircular (mostly elliptical) geometry, or by using anisotropic stress applying parts built into the cross-section of the fiber.

The modal behavior of the lowest-order mode HB fibers under various external deformations is of special interest for sensors and device applications. A number of physical quantities can be measured on the basis of $\mathrm{HB}$ fibers: hydrostatic pressure, strain, vibration, temperature, acoustic wave, etc.

A symmetric deformation effect $(X)$ influences the propagation constant $\beta$ in every mode because of the

\footnotetext{
* corresponding author; e-mail: lesiak@if.pw.edu.pl
}

changes in fiber length $L$ and the refractive indices of the core and the cladding. In a single-mode regime, this leads to changes in the phase difference $\Delta \Phi=\Delta \beta L$ between both polarizations of the fundamental $\mathrm{LP}_{01}$ mode along the fiber [1]:

$$
\frac{\delta(\Delta \Phi)}{\delta X}=\Delta \beta \frac{\partial L}{\partial X}+L \frac{\partial(\Delta \beta)}{\partial X},
$$

where $X$ stands for temperature $(T)$, pressure $(p)$ or longitudinal strain $(\varepsilon)$ defined as $\varepsilon=\Delta L / L$.

The effect of longitudinal strain on mode coupling is to modulate the relative phase retardation between the two orthogonal polarizations in the $\mathrm{LP}_{01}$ mode. The general formula describing the birefringence sensitivity to strain can be expressed in terms of an experimental parameter $T_{\varepsilon}$ describing the amount of strain $\varepsilon$ required to induce a $2 \pi$ phase shift of a polarized light observed at the output as [3]:

$$
\Delta \beta(\varepsilon)=\Delta \beta^{0}+\operatorname{sgn}\left(\frac{\mathrm{d}(\Delta \beta)}{\mathrm{d} \varepsilon}\right) \varepsilon \frac{2 \pi}{T_{\varepsilon} L},
$$

where $\Delta \beta_{L}^{0}$ signifies unperturbed modal (polarization) birefringence of a fiber and the function $\operatorname{sgn}\left(\frac{\mathrm{d}(\Delta \beta)}{\mathrm{d} \varepsilon}\right)$ has two values: +1 or -1 depending on the sign of the 
changes in the relative polarization birefringence with strain and $L$ is the total optical path of the fiber.

Under influence of the longitudinal strain the first term on the right-hand side of expression (1) is negligible with respect to the first, so that

$$
\delta(\Delta \Phi) \cong \frac{\partial(\Delta \beta)}{\partial \varepsilon} L \varepsilon=\frac{\partial(\Delta \beta)}{\partial \varepsilon} \delta L .
$$

Hence the phase changes of the polarimetric responses are proportional to the absolute elongation $\Delta L$ and are independent of the length $L$ of the sensing region. Under the influence of a longitudinal axial strain, Eq. (3) can be approximated with the use of formulae (2) in terms of the only experimental parameter $T_{\varepsilon}$ :

$$
\begin{aligned}
& \frac{\delta\left(\Delta \Phi_{i}\right)}{\delta \varepsilon}=\Delta \beta_{i} \frac{\partial L}{\partial \varepsilon}+\operatorname{sgn} \frac{\mathrm{d}\left(\Delta \beta_{i}\right)}{\mathrm{d} \varepsilon} \frac{2 \pi}{T_{\varepsilon}^{i}} \\
& \cong \operatorname{sgn} \frac{\mathrm{d}\left(\Delta \beta_{i}\right)}{\mathrm{d} \varepsilon} \frac{2 \pi}{T_{\varepsilon}^{i}} .
\end{aligned}
$$

The main advantages of fiber Bragg gratings (FBGs) over other optic sensor schemes are its low cost, good linearity, wavelength multiplexing capacity, resistance in harsh environments and transduction mechanism which eliminates the need for referencing as in interferometric sensors [4-8]. FBG sensor technology is now on the verge of maturity after almost three decades of active research and development in this field. Efforts are now concentrating on delivering complete FBG sensor systems including front-end electronics.

The grating typically has a sinusoidal refractive index variation over a defined length [9]. The reflected wavelength $\left(\lambda_{\mathrm{B}}\right)$, called the Bragg wavelength, is defined by the relationship

$$
\lambda_{\mathrm{B}}=2 n \Lambda,
$$

where $n$ is the effective refractive index of the grating in the fiber core and $\Lambda$ is the grating period.

Fiber Bragg gratings can be used as sensing elements in optical fiber sensors. In a FBG sensor, the measurand causes a shift in the Bragg wavelength, $\Delta \lambda_{\mathrm{B}}$. The relative shift in the Bragg wavelength, $\Delta \lambda_{\mathrm{B}} / \lambda_{\mathrm{B}}$, due to an applied strain $(\varepsilon)$ is approximately given by

$$
\frac{\Delta \lambda_{\mathrm{B}}}{\lambda}=C_{\mathrm{s}} \varepsilon,
$$

where $C_{s}$ is the coefficient of strain.

The stress-induced $(\varepsilon)$ elongation of the sample in the function of the deflection is given by following formulae:

$$
\varepsilon=6 \frac{s d}{L^{2}},
$$

where $s$ - deflection, $d$ - distance between fiber layers, $L$ - length of the sample.

The change of the Bragg reflection in the function of the elongation is $1.2 \mathrm{pm}$ for $1 \mu \varepsilon$ at $1500-1600 \mathrm{~nm}$ [9].

In recent years, many experiments and researches have been carried out on laminated composites to obtain optimal mechanical properties [10-17]. Composite properties are highest in the orientation direction of fibers. In practical application, most of the structures are not loaded in a single direction, it is necessary to orient fibers in multiple directions. This demands evaluation of mechanical properties for different fiber orientations. The fiber weight fraction is an important parameter influencing the mechanical properties of composites.

\section{Materials and measurement system}

We have introduced three fibers inside the composite material separated differently from the central part of the sample. The sample (Fig. 1) was $200 \mathrm{~mm}$ long, $25 \mathrm{~mm}$ wide and $5 \mathrm{~mm}$ thick. The distance between fiber sensors layers was set to $d=0.5 \mathrm{~mm}$. The sample was made at Faculty of Materials Science and Engineering, Warsaw University of Technology.

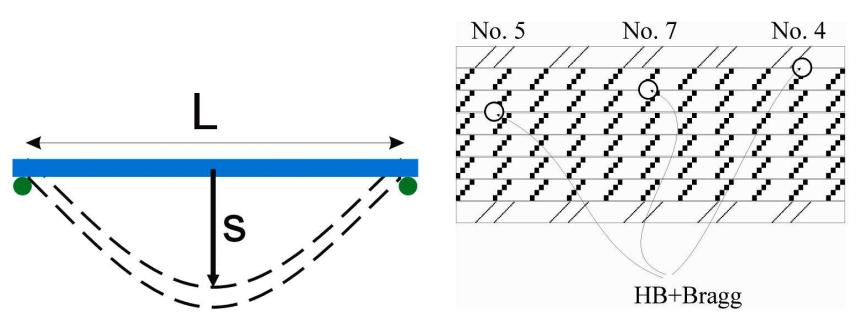

Fig. 1. Scheme of the deflection measurements and fiber optic sensors distribution in the composite material.

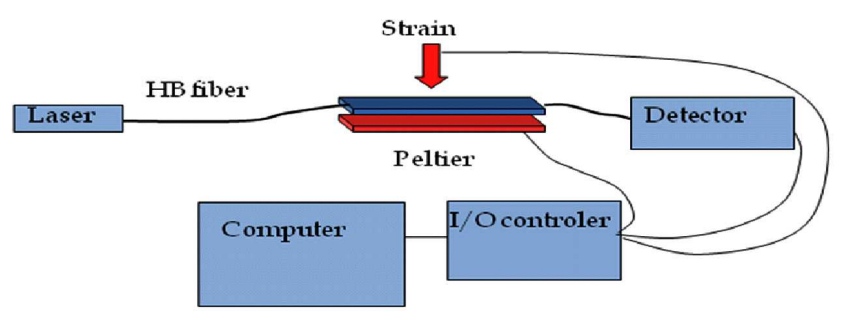

Fig. 2. Measurement setup.

The wavelength and phase changes of the Bragg and polarimetric sensors, respectively, are measured by using a PAT9000B polarimeter. This system (see Fig. 2) allows allows measurement of the Bragg wavelength shift in both polarization axes and additionally phase changes when the analyzer is oriented at $45^{\circ}$ to both axes. Three different fiber optic sensors were distributed in the composite material as shown in Fig. 1. This allows us to measure stress distribution in the composite material. A laser source operated at the wavelength of $1550 \mathrm{~nm}$ was used as the input source. To introduce strain in the composite material sample, the sample was bent by applying force in the middle region of the sample. The average strain experienced by the sample is calculated analytically [8]. The Peltier thermoelectric device for controllable temperature changes was used. 


\section{Measurement results}

For some measurements two Bragg wavelengths are too close to each other, so in the first step two orthogonal polarization axes in the sensing HB fiber have been found and measurements where made separately for three polarizations simultaneously: both the Bragg sensors (polarization 0 and 90 degree is shown in Fig. 3) and the polarimetric sensor (45 degree). When the light was propagating along one polarization axis no temperature sensitivity of the polarimetric fiber sensor was observed and only the Bragg sensors were sensitive to temperature changes.

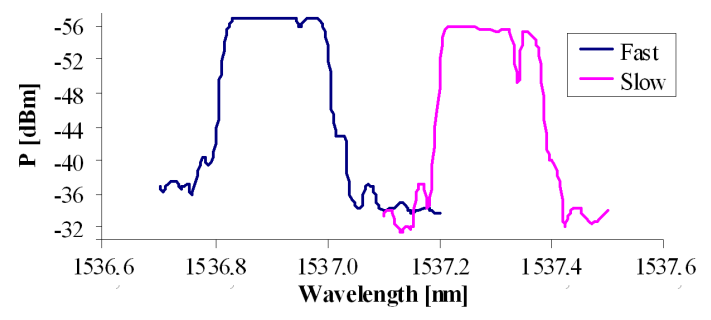

Fig. 3. Typical difference in spectral characteristics of unperturbed fiber sensor No. 7 observed for two orthogonal polarization axes (fast and slow).

As it was shown in our previous papers [18, 19], the sign of the bend-induced phase change reveals different stress mechanisms induced in the composite material. It appeared that position of the sensing fiber (below, in the middle, or above the center of the sample) plays a predominant role in the stress-induced effects. Based on the results reported in this paper, we have demonstrated that the bend-induced phase change strongly depends on the sensor position within the composite sample. As shown in Fig. 4a, the fiber optic sensor system allows one measuring stress distribution in the bent composite material. Stress induced by fiber elongation measured by sensor No. 4 was much higher than stress induced by fiber elongation measured by the sensor No. 5 . The sensors in this composite material were placed in different positions (see Fig. 1) so it was possible that the distance between layers of the composite material could not be uniform in the whole sample. This might explain why we observed small elongation difference between the sensors No. 4 and No. 7. Temperature changes are almost uniform in the whole cross-section of the sample. Results presented in Fig. 4b show similar Bragg wavelength shifts in all three installed FBG-based sensors.

Results obtained for two orthogonal polarizations give additional information about differences between sensor responses to the external perturbation. Since fiber elongation increases birefringence in HB fibers (see Fig. 5b and Eq. (2)) a difference between the Bragg wavelength shifts will also increase for the Bragg sensors (Fig. 4a). Additionally, the dynamics of these changes strongly depends on the sensor distance from the middle of the sample.

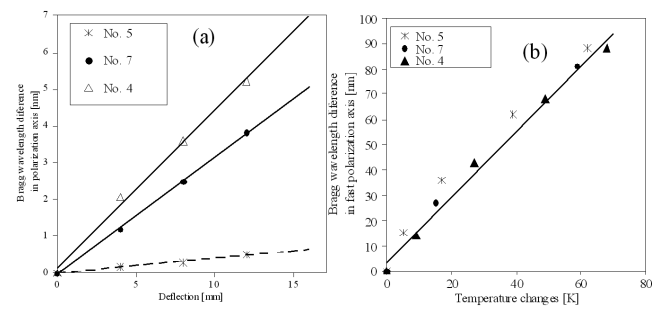

Fig. 4. Measured FBG wavelength shift in fast polarization axis with applied strain (a) and temperature (b).

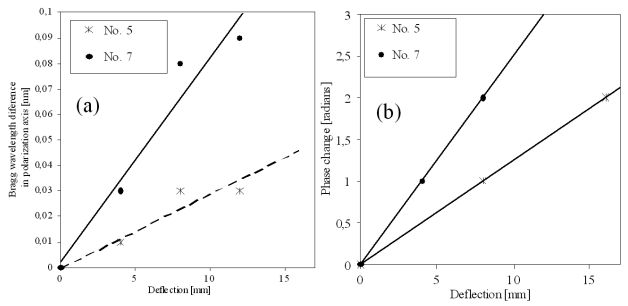

Fig. 5. Measured FBG wavelength shift difference in both polarization axes (a) and birefringence difference (b) with applied strain.

In HB fibers, with stress-induced birefringence measurements show that birefringence decreases in function of temperature (Fig. 6b). In this case the difference between the Bragg wavelengths shift for the FBG sensors (Fig. 6a) decreases. Additionally, dynamics of these changes does not depend on sensor distance from the middle of the sample because temperature is uniform in the cross-section of the composite sample.

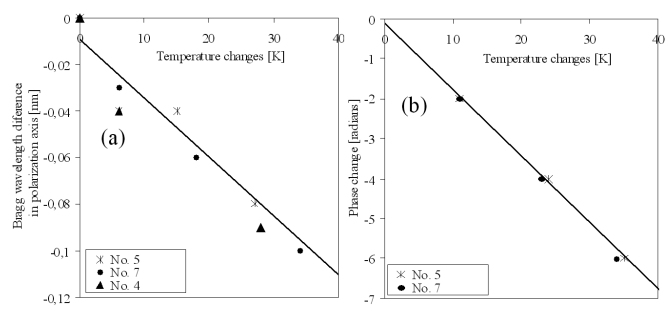

Fig. 6. Measured FBG wavelength shift difference in both polarization axes (a) and birefringence difference (b) with temperature.

\section{Summary}

In this paper new experimental results of the stress distribution inside the composite material measured by FBG sensors written in HB fibers have been presented. The FBG sensors distributed in the composite material reveal information about both stress distribution and temperature and differences in thermal and strain responses of these sensors give the possibility for simultaneous temperature and deformation measurements. 


\section{Acknowledgments}

This work was supported by the Polish Ministry of Science and Higher Education through The National Centre for Research and Development under the grant ERA-NET MATERA.

\section{References}

[1] T.R. Wolinski, in: Progress in Optics, Ed. E. Wolf, Vol. XL, North-Holland, Amsterdam 2000, p. 1.

[2] T.R. Wolinski, in: Enc. Opt. Engineering, Ed. R. Diggers, Marcel Dekker, New York 2003, p. 2150.

[3] W.J. Bock, A.W. Domanski, T.R. Wolinski, Appl. Opt. 29, 3484 (1990).

[4] HD. Simonsen, R. Paetsch, JR Dunphy, Proc. SPIE 1777, 73 (1992).

[5] P. Sivanesan, J. Sirkis, V. Venkat, YC. Shi, C. Reddy, S. Sankaran, Proc. SPIE 3670, 92 (1999).

[6] S. Huang, M. Ohn, M. LeBlanc, R.M. Measures, Smart Mater. Struct. 7, 248 (1008).

[7] Y. Lo, F. Xiao, J. Intellig. Mater. Syst. Struct. 9, 800 (1998).

[8] V. Dewynter-Marty, P. Ferdinand, J. Intellig. Mater. Syst. Struct. 9, 785 (1998).

[9] A. Othonos, K. Kalli, Fiber Bragg Gratings Fundamentals and Applications in Telecommunications and Sensing, Artech House, Norwood (Mass) 1999.
[10] K. Kuang, R. Kenny, M. Whelan, W. Cantwell, P.R. Chalker, Composite Sci. Technol. 61, 1379 (2001).

[11] J. Guemes, J.M. Diaz-Carrilo Menendes, Proc. SPIE 3330, 264 (1998).

[12] J. Guemes, J.M. Diaz-Carrilo Menendes, Composite Sci. Technol. 62, 959 (2002).

[13] G. Reyes, W.J. Cantwell, Composites Sci. Technol. 60, 1085 (2000).

[14] P. Davies, W.J. Cantwell, Composites 25, 869 (1994).

[15] K. Peters, M. Studer, J. Botsis, A. Iocco, H.G. Limberger, R. Salathe, Proc. SPIE 3670, 195 (1999).

[16] T.K. Gangopadhyay, M. Majumder, A.K. Chakraborty, A.K. Dikshit, D.K. Bhattacharya, Sensors Actuators A 150, 78 (2009).

[17] N.C. Eaton, M.J. Curran, J.P. Dakin, H. Geiger, Smart Composites 09, 20 (1993).

[18] A.W. Domanski, P. Lesiak, K. Milenko, D. Budaszewski, M. Chychlowski, S. Ertman, M. Tefelska, T.R. Wolinski, K. Jedrzejewski, L. Lewandowski, W. Jasiewicz, J. Helsztynski, A. Boczkowska, Acta Phys. Pol. A 116, 294 (2009).

[19] A.W. Domański, P. Lesiak, K. Milenko, A. Boczkowska, D. Budaszewski, S. Ertman, T.R. Woliński, Photon. Lett. Poland 1, 121 (2009). 